\title{
Venous Reddening as a Possible Sign of Hyperperfusion after Superficial Temporal Artery-Middle Cerebral Artery Anastomosis for Moyamoya Disease: Case Report
}

\author{
Toshio MACHIDA, ${ }^{1}$ Junichi OnO, ${ }^{1}$ Ryota NOMURA, ${ }^{2}$ Atsushi FUJIKAWA, ${ }^{1}$ \\ Osamu NAGANO, ${ }^{1}$ and Yoshinori HIGUCHI ${ }^{2}$
}

\author{
${ }^{1}$ Department of Neurosurgery, Chiba Cardiovascular Center, Ichihara, Chiba; \\ ${ }^{2}$ Department of Neurosurgery, Chiba University Graduate School of Medicine, Chiba, Chiba
}

\begin{abstract}
Here we report a case of moyamoya disease in which cortical veins reddened after superficial temporal artery (STA) to middle cerebral artery (MCA) anastomosis, following postoperative hyperperfusion. A 37-year-old man with moyamoya disease suffered cerebral infarction in his right hemisphere. Single photon emission computed tomography (SPECT) showed impaired cerebral blood flow (CBF) in both cerebral hemispheres. The patient underwent STA-MCA anastomosis in the right cerebral hemisphere. During operation, soon after declamping the STA, cortical veins near the anastomosis site changed its color from blue to red. This change was repeatable by clamping and declamping of the STA. Postoperative SPECT and computed tomography (CT) demonstrated increased CBF and subarachnoid hemorrhage at the anastomosis site, suggesting the occurrence of postoperative hyperperfusion. By strictly controlling the patient's blood pressure, the syndrome resolved 1 week after the operation. We propose that the venous reddening after STA-MCA anastomosis may be a sign of postoperative hyperperfusion.
\end{abstract}

Key words: venous reddening, hyperperfusion, moyamoya disease, superficial temporal artery-middle cerebral artery anastomosis

\section{Introduction}

Superficial temporal artery (STA) to middle cerebral artery (MCA) anastomosis is an effective surgical procedure for the treatment of moyamoya disease. ${ }^{1-3)}$ Compared to indirect bypass surgeries, such as encephalo-duro-arterio-synangiosis and encephalo-myo-synangiosis, this procedure has the advantage of rapid improvement of impaired cerebral blood flow (CBF) and remission of ischemic symptoms. In spite of immediate effects, recent studies have revealed that a considerable number of patients (15\% to $50 \%)$ suffer hyperperfusion syndrome after the operation. ${ }^{4-8)}$ Because the syndrome may lead to permanent neurological deficits without prompt blood pressure control, ${ }^{4,5)}$ anticipation of the syndrome is critical.

Recently, some investigators have attempted to predict hyperperfusion syndrome during the surgery by use of specialized equipments such as laser Doppler flowmetry and thermography. ${ }^{9,10)}$ However, the diagnosis of the syndrome has been mainly based on a postoperative

Received August 6, 2013; Accepted December 2, 2013 radiological examination such as SPECT,,-8) and little has been known about morphological change which associates with hyperperfusion. Here, we report a case of moyamoya disease in a man whose cortical veins reddened soon after STA-MCA anastomosis. After the operation he developed hyperperfusion syndrome. We propose that the reddening may be a sign of postoperative hyperperfusion and may contribute to further understanding of the pathomechanisms of hyperperfusion.

\section{Case Report}

A 37-year-old man experienced transient weakness and sensory disturbance in his right arm and leg. Two weeks later, he suffered right hemiparesis and visited a neurosurgical clinic nearby. Magnetic resonance imaging (MRI) demonstrated cerebral infarction in his left frontal and parietal lobes and occlusion of the terminal portion of the bilateral internal carotid artery (Fig. 1A, B). He was referred to our hospital for further evaluation and treatment. Digital subtraction angiography showed an occlusion of the terminal portion of the bilateral internal 
carotid artery and moyamoya vessels in the basal ganglia. The diagnosis was stage III moyamoya disease according to the criteria of the Research Committee on Spontaneous Occlusion of the Circle of Willis of the Ministry of Health, Labor, and Welfare, Japan. ${ }^{3)}$ Single-photon emission computed tomography with $\mathrm{N}$-isopropyl[123I]p-iodoamphetamine (IMP-SPECT) with and without acetazolamide showed marked decrease in cerebral blood flow (CBF) and impaired cerebral vascular reserve capacity (CVRC) in both cerebral hemispheres (Fig. 1C, D). The impaired CBF due to moyamoya disease was considered to be the cause of the cerebral infarction. We scheduled revascularization surgery for his left hemisphere. However, while waiting for the surgery, the patient suffered from a common cold, which was followed by new cerebral infarction in his right temporal and occipital lobes (Fig. 1E).

After 1 month of medical treatment, STA-MCA anastomosis with encephalo-myo-synangiosis in his right cerebral hemisphere was performed under general anesthesia using propofol and fentanyl. During surgery, systolic blood pressure and arterial carbon dioxide pressure was maintained at $100-140 \mathrm{mmHg}$ and $38-42 \mathrm{mmHg}$, respectively. After the exposure of the cortical branch of the MCA (M4) on the temporal lobe (Fig. 2A), the recipient artery was temporarily interrupted and the parietal branch of the STA was anastomosed in an end to side fashion (Fig. 2B). The total interrupting time of the recipient artery was 22 minutes A few seconds after declamping the STA, the cortical veins near the anastomosis site gradually changed its color from blue to red (Fig. 2B) and the arterioles around the anastomosis site dilated. This phenomenon was repeated by clamping and declamping the STA.

The patient recovered from anesthesia without any new neurological symptoms and his systolic blood pressure was strictly controlled between $100 \mathrm{mmHg}$ and $140 \mathrm{mmHg}$ to avoid both ischemic and hemorrhagic complications. CT scan, the day after the operation, showed no new lesions (Fig. 3A). However, SPECT two days after the operation demonstrated marked increase in CBF around the anastomosis site in his right temporal lobe (Fig. 3C) and CT scan three days after the operation showed subarachnoid hemorrhage in the same region (Fig. 3B). Due to the diagnosis of postoperative hyperperfusion, we strictly controlled his systolic blood pressure to a normal range (100-130 mmHg). He did not complain of any new symptoms and follow-up SPECT eight days after the operation demonstrated normalized CBF in his right cerebral hemisphere indicating the remission of the syndrome (Fig. 3D). MRA 14 days after the operation
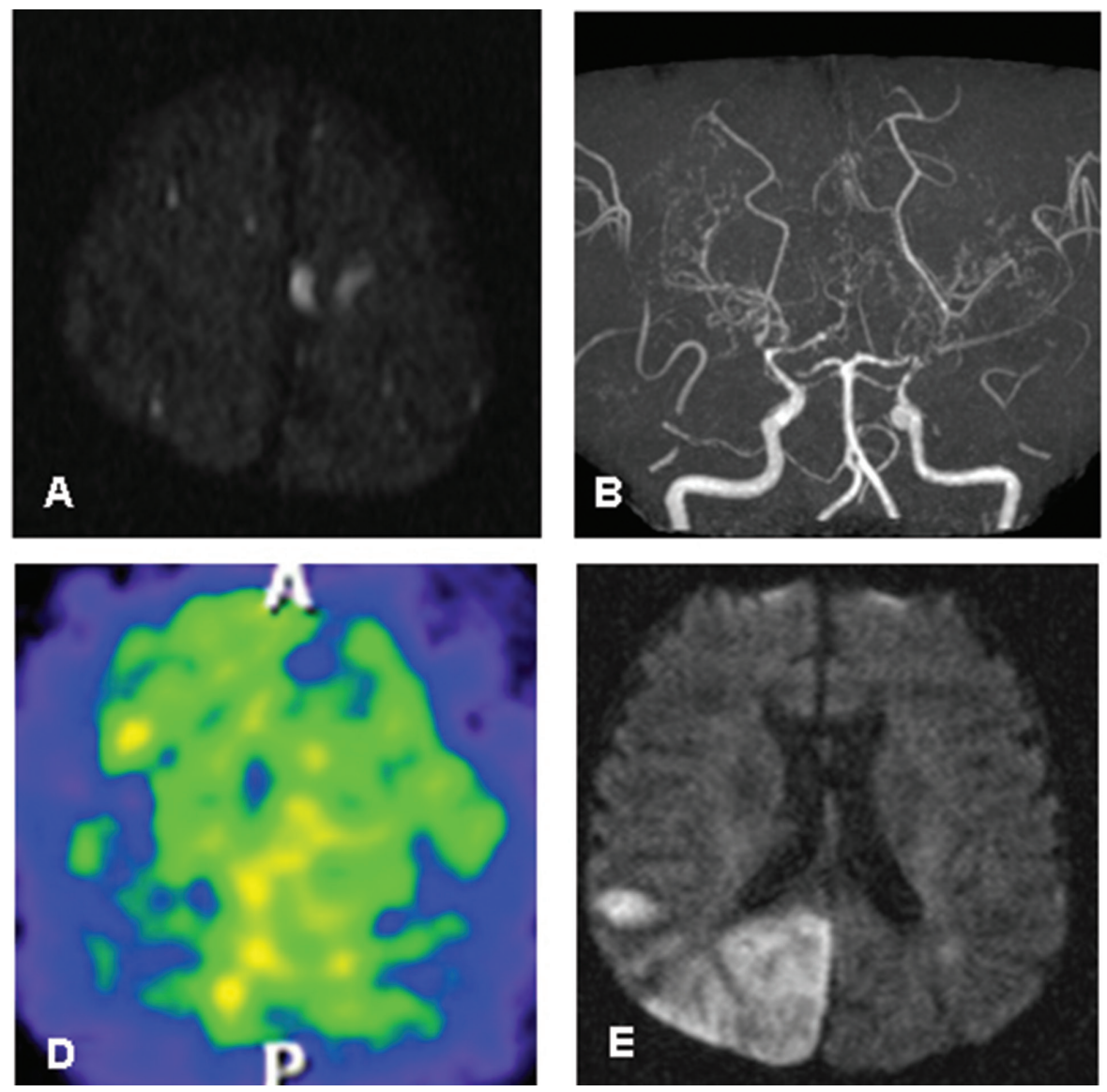

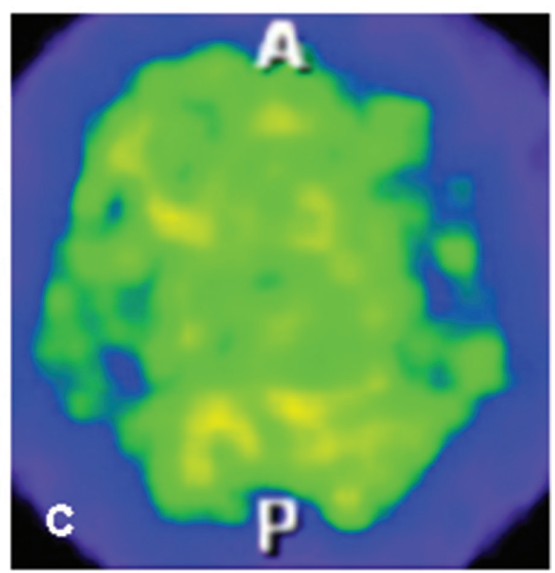

Fig. 1 A: Diffusion weighted imaging (DWI) during the initial attack showing small cerebral infarctions in both cerebral hemispheres. B: Magnetic resonance angiography showing occlusion of the terminal portion of the internal carotid artery bilaterally and moyamoya vessels. C, D: Preoperative single-photon emission computed tomography with (C) and without (D) acetazolamide showing decreased cerebral blood flow and impaired vascular reserve capacity in both cerebral hemispheres. E: DWI after the second attack showing cerebral infarction in the right parietal and posterior cerebral lobe. A: anterior, P: posterior. 


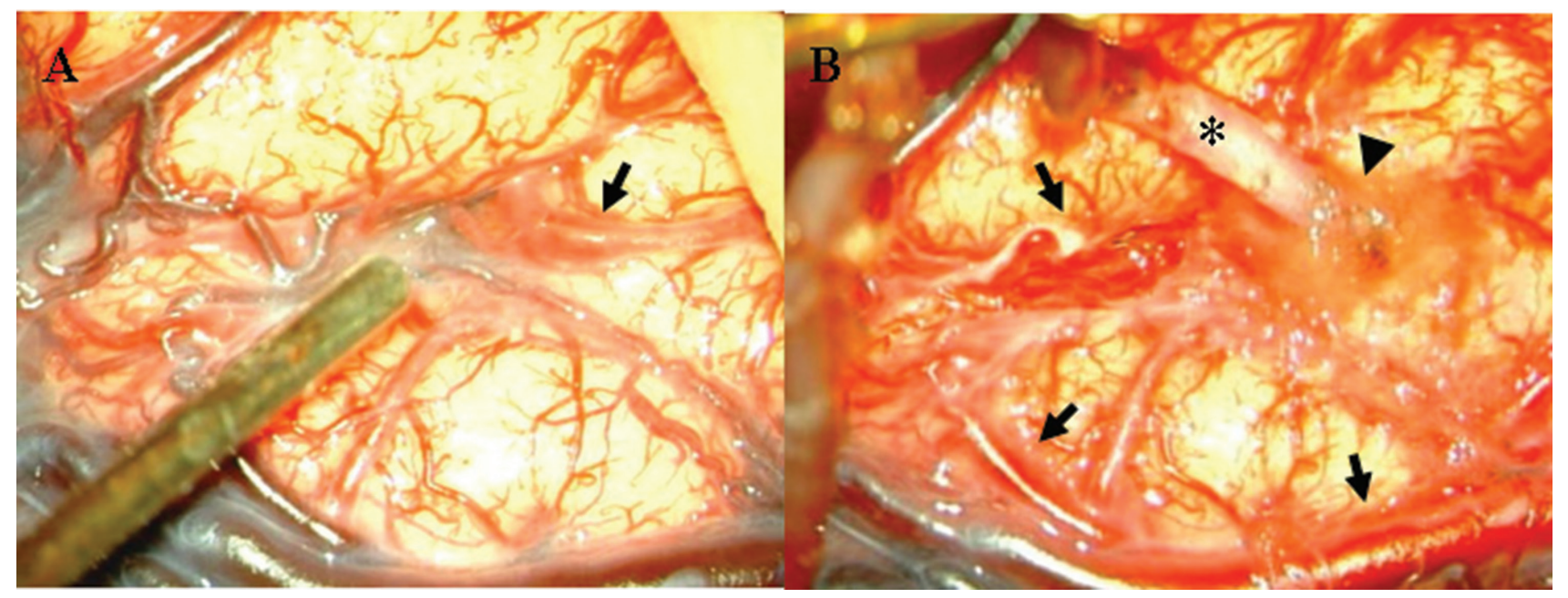

Fig. 2 A: Before the anastomosis, the cortical branch (M4) of the middle cerebral artery on the temporal lobe (arrow) was exposed. B: Photograph at 5 minutes after declamping the superficial temporal artery (STA). Parietal branch of the STA (asterisk) was anastomosed to the recipient in an end to side fashion (arrowhead). Cortical veins near the anastomosis site reddened a few seconds after declamping the STA (arrows) and the dilated arterioles are also observed.
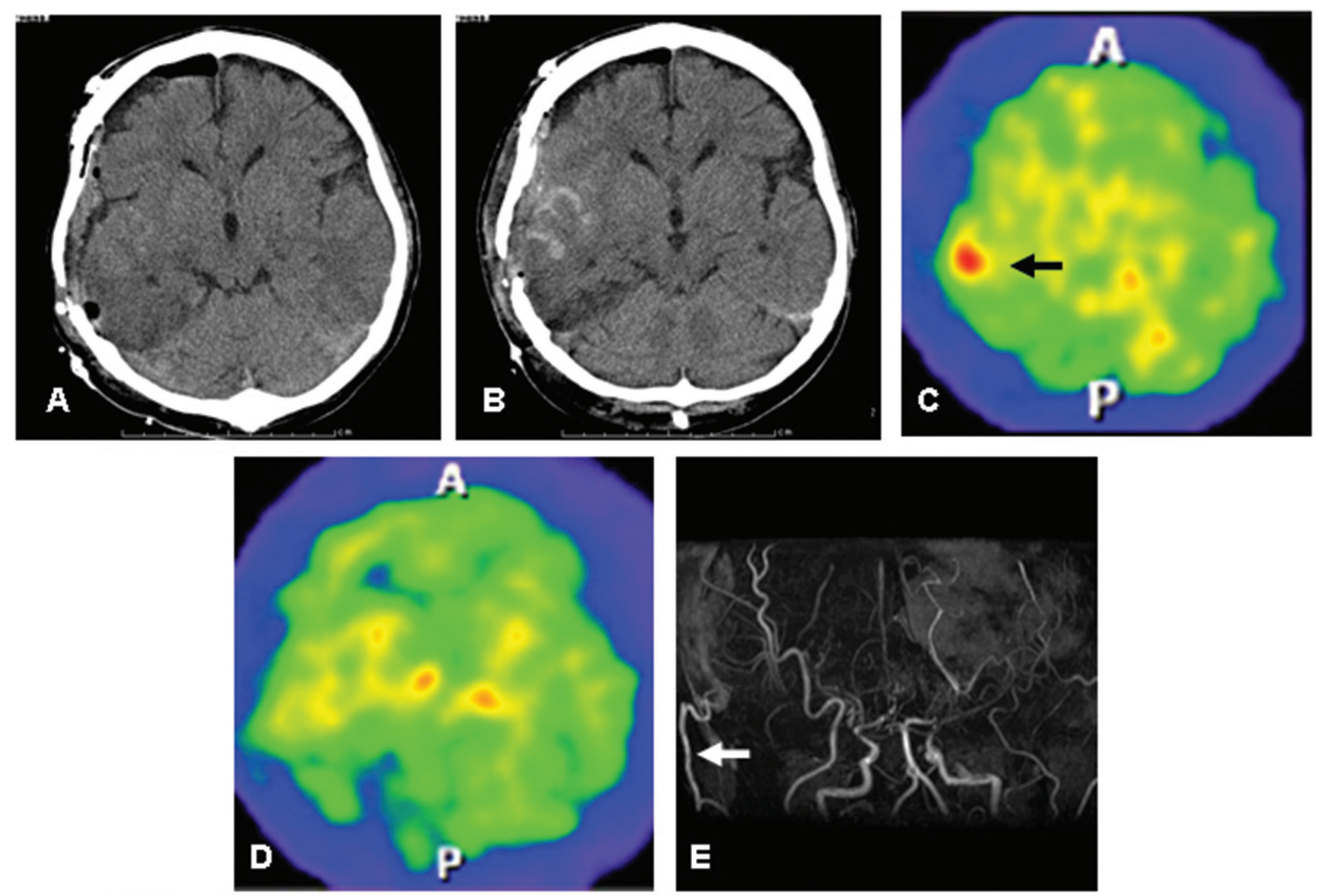

Fig. 3 A: Computed tomography (CT) scan one day after operation showing no new lesions. B: CT scan 3 days after operation showing subarachnoid hematoma at the anastomosis site. C: Single-photon emission computed tomography (SPECT) 2 days after the operation demonstrating increased cerebral blood flow (CBF) at the anastomosis site (arrow) D: Follow-up SPECT 8 days after the operation showing mildly increased CBF in the right cerebral hemisphere and disappearance of local hyperperfusion. E: Magnetic resonance angiogram 14 days after the operation showing well-developed STA (arrow). A: anterior, $\mathrm{P}$ : posterior. 
demonstrated a well-developed STA (Fig. 3E).

\section{Discussion}

Postoperative hyperperfusion is a serious complication after revascularization surgery for steno-occlusive cerebrovascular diseases. ${ }^{6,7)}$ Hyperperfusion after carotid endarterectomy (CEA) and carotid angioplasty (CAS) are well recognized pathological conditions. ${ }^{11,12)}$ The incidence of the syndrome is relatively low ( $0 \%$ to $3 \%$ ) but it may cause critical complications, such as intracerebral hemorrhage and brain edema, leading to severe neurological deterioration. $^{6,12-14)}$ The reductions of preoperative CBF and CVRC are known as the significant risk factors for hyperperfusion and it is suggested that the reduction of preoperative perfusion pressure may induce persistent vasodilatation of the arterioles even after the recovery of perfusion pressure. ${ }^{11,13,14)}$ As a result, large amount of blood flows into the brain tissue and causes serious clinical conditions such as intracerebral hemorrhage and cerebral edema. As to moyamoya disease, because the preoperative reduction of $\mathrm{CBF}$ and CVRC are also reported to be the risk factors for the development of postoperative hyperperfusion, ${ }^{4-8)}$ the pathomechanism of hyperperfusion in moyamoya disease is considered to be similar to that of CEA and CAS.

What then might be the pathomechanism of the venous reddening? The venous color reflects the venous oxyhemoglobin content and, theoretically, the venous oxyhemoglobin content is determined by the difference between the supply and consumption of oxygen in the tissue. During STA-MCA anastomosis, because the oxygen consumption would have been kept constant under general anesthesia, the venous reddening would be caused by an increase in oxygen supply in the tissue. In the present case, marked reductions of preoperative CBF and CVR suggest the preexistence of pathologically dilated arterioles. And, after STA-MCA anastomosis, a large amount of blood may have rushed into the dilated arterioles and supplied excess amounts of oxygen to the tissue. As a result, differences between oxygen supply and consumption in the tissue may have increased leading to an increase in venous oxyhemoglobin content and venous reddening. Recent study using positron emission tomography (PET) demonstrated the change in tissue oxygen metabolism by STA-MCA anastomosis. ${ }^{7)}$ According to the study, oxygen extraction fraction (OEF) decreased by bypass surgery in patients with moyamoya disease who developed hyperperfusion syndrome. Because the decreased OEF indicates a relative increase in oxygen supply compared to the consumption, their finding supports our causal hypothesis of the venous reddening during hyperperfusion.

To our knowledge, little has been known whether hyperperfusion causes visible changes on the brain surface. In this case, we also observed dilation of the arteriole around the anastomosis site. Recent studies using thermography demonstrated that the cortical surface temperature around the anastomosis site highly elevated in patients with hyperperfusion. ${ }^{9,10,15)}$ Because the dilation of the arteriole may contribute to the elevation of brain surface temperature, the dilation may be one of the signs of hyperperfusion that directly reflects the increased CBF. On the other hand, because the degree of venous reddening will be determined by a balance between oxygen supply and consumption in the tissue, the reddening may be another sign of hyperperfusion that represents the altered oxygen metabolism under the pathological condition. Further studies are necessary to improve our understanding of venous reddening, however, we propose that this phenomenon may contribute to our understanding of the pathomechanisms underlying hyperperfusion.

\section{Conflicts of Interest Disclosure}

The authors report no conflicts of interest concerning the materials or methods used in this study or the findings specified in this article. All authors are the members of the Japan Neurosurgical Society (JNS) and have registered online Self-reported COI Disclosure Statement Forms through the website for JNS members.

\section{References}

1) Kuroda S, Houkin K: Bypass surgery for moyamoya disease: concept and essence of surgical techniques. Neurol Med Chir (Tokyo) 52: 287-294, 2012

2) Kuroda S, Houkin K: Moyamoya disease: current concepts and future perspectives. Lancet Neurol 7: 1056-1066, 2008

3) Research Committee on the Pathology and Treatment of Spontaneous Occlusion of the Circle of Willis: Health Labour Sciences Research Grant for Research on Measures for Infractable Diseases. Guidelines for diagnosis and treatment of moyamoya disease (Spontaneous Occlusion of the Circle of Willis). Neurol Med Chir (Tokyo) 52: 245-266, 2012

4) Fujimura M, Inoue T, Shimizu H, Saito A, Mugikura S, Tominaga T: Efficacy of prophylactic blood pressure lowering according to a standardized postoperative management protocol to prevent symptomatic cerebral hyperperfusion after direct revascularization surgery for moyamoya disease. Cerebrovasc Dis 33: 436-445, 2012

5) Fujimura M, Mugikura S, Kaneta T, Shimizu H, Tominaga $\mathrm{T}$ : Incidence and risk factors for symptomatic cerebral hyperperfusion after superficial temporal artery-middle cerebral artery anastomosis in patients with moyamoya disease. Surg Neurol 71: 442-447, 2009

6) Hayashi K, Horie N, Suyama K, Nagata I: Incidence and clinical features of symptomatic cerebral hyperperfusion syndrome after vascular reconstruction. World Neurosurg 78: 447-454, 2012

7) Kaku Y, Iihara K, Nakajima N, Kataoka H, Fukuda K, Masuoka J, 
Fukushima K, Iida H, Hashimoto N: Cerebral blood flow and metabolism of hyperperfusion after cerebral revascularization in patients with moyamoya disease. J Cereb Blood Flow Metab 32: 2066-2075, 2012

8) Uchino H, Kuroda S, Hirata K, Shiga T, Houkin K, Tamaki $\mathrm{N}$ : Predictors and clinical features of postoperative hyperperfusion after surgical revascularization for moyamoya disease: a serial single photon emission CT/positron emission tomography study. Stroke 43: 2610-2616, 2012

9) Kawamata T, Kawashima A, Yamaguchi K, Hori T, Okada Y: Usefulness of intraoperative laser Doppler flowmetry and thermography to predict a risk of postoperative hyperperfusion after superficial temporal artery-middle cerebral artery bypass for moyamoya disease. Neurosurg Rev 34: $355-362,2011$

10) Nakagawa A, Fujimura $M$, Arafune $T$, Sakuma I, Tominaga $\mathrm{T}$ : Clinical implications of intraoperative infrared brain surface monitoring during superficial temporal artery-middle cerebral artery anastomosis in patients with moyamoya disease. J Neurosurg 111: 1158-1164, 2009

11) Abou-Chebl A, Yadav JS, Reginelli JP, Bajzer C, Bhatt D, Krieger DW: Intracranial hemorrhage and hyperperfusion syndrome following carotid artery stenting: risk factors, prevention, and treatment. J Am Coll Cardiol 43: 1596-1601, 2004

12) Ogasawara K, Sakai N, Kuroiwa T, Hosoda K, Iihara K,
Toyoda K, Sakai C, Nagata I, Ogawa A; Japanese Society for Treatment at Neck in Cerebrovascular Disease Study Group: Intracranial hemorrhage associated with cerebral hyperperfusion syndrome following carotid endarterectomy and carotid artery stenting: retrospective review of 4494 patients. J Neurosurg 107: 1130-1136, 2007

13) Ogasawara K, Yukawa H, Kobayashi M, Mikami C, Konno H, Terasaki K, Inoue T, Ogawa A: Prediction and monitoring of cerebral hyperperfusion after carotid endarterectomy by using single-photon emission computerized tomography scanning. J Neurosurg 99: 504-510, 2003

14) van Mook WN, Rennenberg RJ, Schurink GW, van Oostenbrugge RJ, Mess WH, Hofman PA, de Leeuw PW: Cerebral hyperperfusion syndrome. Lancet Neurol 4: 877-888, 2005

15) Okada $Y$, Kawamata T, Kawashima A, Hori T: Intraoperative application of thermography in extracranial-intracranial bypass surgery. Neurosurgery 60(4 Suppl 2): 362-365, 2007

Address reprint requests to: Toshio Machida, MD, PhD, Department of Neurosurgery, Chiba Cardiovascular Center, 575 Tsurumai, Ichihara, Chiba 290-0512, Japan. e-mail: tmachida@ca2.so-net.ne.jp 